\title{
Comparison of scattering by different nonspherical, wavelength-scale particles
}

\author{
Timo Nousiainen ${ }^{\mathrm{a}, *}$, Evgenij Zubko ${ }^{\mathrm{a}}$, Hannakaisa Lindqvist ${ }^{\mathrm{a}}$, Michael \\ Kahnert $^{\mathrm{b}}$, Jani Tyyneläa \\ ${ }^{a}$ Department of Physics, P.O.Box 48, FI-00014 University of Helsinki, Finland \\ ${ }^{b}$ Swedish Meteorological and Hydrological Institute, Folkborgsvägen 1, 60176 Norrköping, \\ Sweden
}

\begin{abstract}
It is well established that spherical and nonspherical particles scatter light differently. There are a large number of studies where scattering properties of different nonspherical particles are studied. Here we study to what degree scattering matrices of different nonspherical particles resembles each other, and whether there are significant correlations between morphological similarity and similar single-scattering properties. Altogether 15 different shapes are considered, including both irregular and regular shapes as well as homogeneous and inhomogeneous particles. For all nonspherical particles, orientation- and ensemble-averaged scattering properties are considered, and variability within each ensemble is ignored. The results reveal that different nonspherical shapes have surprisingly similar phase functions. An analysis of the asymmetry parameter reveals that the resemblance is, however, only qualitative: the phase functions are featureless and predominantly flat at side scattering, but they are nevertheless different. The degree of linear po-
\end{abstract}

\footnotetext{
*Corresponding author

Email address: timo.nousiainen@helsinki.fi (Timo Nousiainen)
} 
larization for unpolarized incident light shows much larger differences among the shapes, albeit it is much more positive for all nonspherical targets than for Mie spheres. Similarly to the phase function, the depolarization ratio tends to be similar among the nonspherical particle types, implying that the strength of depolarization cannot be used as a measure for the degree of nonsphericity. In general, it is found that there does not seem to be a clear correlation between particle morphology and scattering properties.

\section{Introduction}

One of the main objectives in the field of light scattering by particles is to understand how physical properties, i.e. the size, morphology, and dielectric properties, are related to the particles' optical properties. This question is particularly important in the context of inverse modelling and remote sensing. The earliest studies in the field focused on light scattering by homogeneous spheres [1], for which the light-scattering problem can be solved analytically. The homogeneous sphere model can only account for a particle's size and effective dielectric properties, but it neglects more complex morphological features, such as non-sphericity, aggregation, or inhomogeneity. Thus this model is only capable of reproducing optical characteristics that are mainly size dependent and largely insensitive to particle morphology. For instance, for some non-spherical homogeneous particles, the width of the forward-peak of the phase function, as well as the single-scattering albedo are mainly sizedependent and rather insensitive to particle shape [2]. In general, however, the polarized differential scattering properties are highly sensitive to particle morphology. 
The use of more sophisticated model geometries is complicated by both practical and technical challenges. It is technically difficult to use model geometries with a high degree of morphological details, owing to high CPU time requirements or ill-conditioning problems in numerical light-scattering computations. Despite such difficulties, much progress has been made in recent years in the development of numerical solvers for light scattering problems (see [3] and references therein), which has paved the way for studies of more realistic model geometries. However, it is practically not possible to employ exact replicas of each and every particle shape encountered in nature. Therefore, one often devises model geometries that emphasise certain morphological features, such as aggregation [4-6], irregularity [7], small-scale surface roughness [8-10], surface dusting [11], or inhomogeneity [12]. To test the suitability of different model geometries for operational applications in remote sensing and climate modelling, one can compare computed optical properties with laboratory measurements (see, e.g., [13] and references therein). On a more fundamental level, one can gain significant empirical insight into the interrelation of physical and optical particle properties by performing numerical experiments. One approach is to develop parametrized models of particle geometries, and to investigate the changes in the optical properties as one varies the geometric parameters [7, 14-16]. Another approach is to intercompare computed optical properties of model particles with different degrees of sophistication. Such intercomparisons have often focused on certain morphological aspects, such as different degrees of geometric symmetries [17], small-scale surface roughness [18], or differences due to regular and stochastic particle geometries [19]. 
In the present study we perform a comprehensive intercomparison of light-scattering properties of 15 different shapes ranging from homogeneous spheres to highly irregular and inhomogeneous scatterers. For all nonspherical targets, scattering is averaged over an ensemble and over orientations. Our main objectives are to establish what are the similarities and differences in scattering among such diverse classes of shapes; and whether scattering correlates in any clear way with the morphology of the scatterers. The study is largely motivated by the earlier findings that scattering by irregularly shaped, rough, and potentially inhomogeneous real dust particles can be mimicked surprisingly well by ensembles of simple spheroids, ellipsoids, or nonsymmetric hexahedra $[17,20,21]$; and by the fact that the shapes within these well-fitting ensembles may not necessarily resemble the target particles in any clear way [22]. To mitigate the otherwise quite considerable computational requirements, we use, whenever available, existing data of particle optical properties.

\section{Theoretical aspects}

When the properties of light, or more generally those of any electromagnetic radiation, are described by the Stokes parameters [23], the properties of incident and scattered light are connected by a $4 \times 4$ Mueller matrix. There are varying definitions for such matrices, depending on their normalization; 
here we use the scattering matrix $\mathbf{S}$ [23], defined such that

$$
\left(\begin{array}{c}
I_{\mathrm{s}} \\
Q_{\mathrm{s}} \\
U_{\mathrm{s}} \\
V_{\mathrm{s}}
\end{array}\right)=\frac{1}{k^{2} d^{2}}\left[\begin{array}{cccc}
S_{11} & S_{12} & S_{13} & S_{14} \\
S_{21} & S_{22} & S_{23} & S_{24} \\
S_{31} & S_{32} & S_{33} & S_{34} \\
S_{41} & S_{42} & S_{43} & S_{44}
\end{array}\right]\left(\begin{array}{c}
I_{\mathrm{i}} \\
Q_{\mathrm{i}} \\
U_{\mathrm{i}} \\
V_{\mathrm{i}}
\end{array}\right)
$$

where $I, Q, U$, and $V$ are the Stokes parameters; $k$ is the wave number $k=2 \pi / \lambda$, where $\lambda$ is the wavelength; $d$ is the distance from the particle to the observer; and subscripts 's' and 'i' refer to scattered and incident radiation, respectively. For incoherent scattering, the Stokes parameters, and thus also the scattering matrix $\mathbf{S}$, are additive.

The scattering matrix elements contain information about the scatterer and depend on the wavelength as well as the (complex) refractive index $m$, shape, size, and orientation of the scatterer. In addition, they are functions of the scattering angle $\theta$ that specifies the angle between the propagation directions of incident and scattered radiation and the azimuth angle $\phi$; the latter dependence vanishes for random orientation. For simplicity, we only considered the following matrix elements here: the intensity of the scattered light for unpolarized incident light $S_{11}$; the linear polarization of scattered light for unpolarized incident light $-S_{12} / S_{11}$; the depolarization ratio $D=$ $1-S_{22} / S_{11}$; and the difference $R=S_{33} / S_{11}-S_{44} / S_{11}$. The last two are indicators for anisotropy in the scatterer, most often due to non-spherical shape, as for isotropic spheres $S_{22}=S_{11}$ and $S_{33}=S_{44}$.

We also consider a number of other quantities derived from the scattering matrix. The asymmetry parameter $g$ is an integral of a normalized $S_{11}$ element and describes the partitioning of the scattered intensity between 
forward and backward hemispheres. It is of particular interest in radiative balance considerations. Scattered intensity and depolarization at backscattering direction, $S_{11}(180)$ and $D(180)$, respectively, are relevant e.g. for lidar applications. The linear and circular polarization ratios, $\mu_{L}$ and $\mu_{C}$, respectively, are defined as

$$
\begin{aligned}
\mu_{L} & =\frac{S_{11}-S_{22}}{S_{11}+2 S_{12}+S_{22}}, \\
\mu_{C} & =\frac{S_{11}+S_{44}}{S_{11}-S_{44}}
\end{aligned}
$$

and are used, e.g., in radioastronomy to characterize different targets. Additionally, we consider the maximum of positive polarization, $\max \left(-S_{12} / S_{11}\right)$ and the scattering angle $\theta_{p}$ at which it is obtained, as well as the amplitude of the negative polarization close to backscattering, $\min \left[-S_{12}(135\right.$ : $\left.180) / S_{11}(135: 180)\right]$.

All our quantities of interest are dimensionless, so they follow the scale invariance rule [24, page 147] and thus depend on the refractive index and the so-called size parameter. The latter conveniently combines the wavelength and size into a single parameter, defined as

$$
x=\frac{2 \pi r}{\lambda},
$$

where $r$ is the radius of a scatterer. In case of non-spherical targets, some kind of equivalent size needs to be established. Here, as described in Section 3, several different types of size equivalences have been used.

\section{Model shapes}

Example images of the shapes considered here are presented in Fig. 1. The parameters with which their single-scattering properties have been computed 
are summarized in Table 1. In the subsections below, the shapes are shortly introduced, but the reader is directed to the original publications for full details on the shape generation and light-scattering simulations. For some shapes, the scattering computations have been carried out specifically for the present study. These are indicated by '-' in the table. For other shapes, computations carried out for the papers indicated are used.

We compare computational results with a refractive index $m$ similar to that of silicate minerals at visible wavelengths, as computations with such values were readily available for many different shapes. As can be seen from the table, the variation in the refractive index among the particle types is generally rather small. In the computations we compare here, the real part of the refractive index is between 1.55 and 1.603. Such a small range will not cause a high impact on scattering that it would influence our mostly qualitative analysis. The imaginary part varies from zero to 0.002. The size parameters of the targets considered are fairly small, so the impact of the variation in $\operatorname{Im}(m)$ is unlikely to have a significant effect on the light scattering properties.

One also needs to keep in mind that comparisons of optical properties of different particle shapes depends on the kind of size measure one employs for different types of particles. As can be seen in the table, different size measure have been adopted for different geometries. We therefore focus in our analysis on general size trends of optical properties within each shape class, rather than performing direct shape-to-shape comparisons at specific sizes. 


\subsection{Modified spheres and agglomerated debris particles}

Five types of irregularly shaped particles, which are referred in Table 1 to as rough-surface sphere, pocked spheres, strongly damaged spheres, debris of spheres, and agglomerated debris particles, have been generated using the same algorithm: First, a spherical volume is defined in a regular cubic lattice that consists of 137,376 cells. The cells are divided into surface and core cells, the depth of the surface layer being a free parameter. For agglomerated debris particles and rough-surface spheres, the depth is only $0.5 \%$ of the total radius, so that the surface layer is formed only by cells having direct contact with the surrounding medium. In the case of pocked spheres, the depth is $12.5 \%$ of the radius. Strongly damaged spheres and debris of spheres are generated having no surface layer, so all cells belong to the core.

Randomly located seed cells are then introduced to the volume. There are two types, one for the empty space and one for material. We further distinguish seed cells for empty space between those within the surface layer and those within the core, while seed cells for the material can only be located in the core. Agglomerated debris particles are generated with 100 seed cells of empty space within the surface layer, and 20 seed cells of empty space and 21 seed cells of material within the core. For rough-surface spheres, the corresponding numbers are 1200, 150, and 0, and for the pocked spheres they are 100, 50, and 0, respectively. For strongly damaged spheres and debris of spheres, the depth of the surface layer and thus also the number of seed cells within it are zero. For strongly damaged spheres, there are 20 seed cells of empty space and 21 seed cells of material for the core; whereas, for debris of spheres both numbers are 4 . Once all the seeds are allocated, each cell in 
the volume is assigned the same properties (refractive index of the material or the empty space) as the nearest seed cell.

The light scattering computations have been performed using a discrete dipole approximation (DDA). In the computations, $m=1.6+0.0005 i$ has been used, and size parameters have been specified based on the size of the circumscribing sphere. The results are ensemble averaged over 200-900 particles and over 4 arbitrary orientations for each particle. For further details, see $[25,26]$.

\subsection{GRS and Rough GRS}

Gaussian random sphere (GRS) is a statistical shape model introduced by [7]. GRS particles are spheres with a deformation in form of a spherical harmonics expansion. To guarantee that the deformation does not produce radii with negative values, the deformation is applied to the logarithm of the radius rather than the radius itself. GRS particles are generated by randomly assigning the weights of the spherical harmonics expansion using statistics specified by the desired autocovariance function of the radius. The GRS particles considered here are based on a power-law parameterization of the autocovariance function with shape parameters $\sigma=0.245$ and $\nu=4$ [27].

The rough GRS particles are generated through a random disturbance of surface layer of initially smooth GRS particles. The surface layer is determined the same way as for the shapes in Section 3.1, using a thickness of five cells. Among surface dipoles, we randomly choose 300 seed cells for both empty space and material. Then, each cell within the surface layer is assigned the same properties as the nearest seed cell. Finally, the size of the rough GRS particle is adjusted such that its volume is the same for the 
original GRS shape. As the rough GRS particle has fewer material cells than the original, its actual size parameter is slightly larger than its nominal size parameter based on the circumscribing sphere of the original GRS particle.

The light scattering computations have been carried out for an ensemble of 100 particles for both the GRS and rough GRS shapes, using the DDA. At least 5 random orientations have been used for each particle in the ensemble, the number increasing until a desired accuracy is reached. A refractive index of $1.6+0.0005 i$ has been used for both shapes.

\subsection{Calcite and clay flakes}

Two different flakes are considered, differing only by their composition (the shapes are identical). One type is assumed to be composed of calcite and is thus birefringent with the ordinary refractive index of $1.658+0.0 i$ and extraordinary refractive index of $1.486+0.0 i$. Such particles are referred to as calcite flakes. The second type, called clay flakes, are isotropic and have a refractive index of $1.603+0.0 i$. The single-scattering data for both types have been published by [28]. The shape of these particles are generated similarly to those of agglomerated debris particles. The main difference is that, instead of a spherical volume, an oblate spheroid with an aspect ratio of 8:1 is used. The resulting shape is also smoothed by removing such volume elements from the particle surface that do not have sufficient number of neighboring volume elements. This procedure is repeated several times to achieve the desired effect. The resulting shapes are irregular, roundish, platy, and have an aspect ratio of about six. The details of the shape generation can also be found from [28]. The single-scattering properties of both flakes have been computed assuming volume-equivalent size parameter, using the DDA. The 
size parameters based on equivalent circumscribing spheres would be about twice as large. The results are averaged over 10 randomly generated sample particles and 729-3375 orientations, depending on the size parameter.

\subsection{Distribution of spheroids}

For spheroids, we consider an ensemble consisting of varying aspect ratios. Let $a$ denote the semi-axis length along the spheroid's main rotational symmetry axis, and $b$ denote the semi-axis length in the perpendicular direction. Then the aspect ratio $\epsilon=b / a$ is smaller than 1 for prolate, and larger than 1 for oblate spheroids. Our ensemble consists of a distribution of 21 aspect ratios ranging from $1 / 3$ to 3 (these extreme cases are shown in Fig. 1). The size parameter $x$ for spheroids is defined such that $x=k a$ for prolate and $x=k b$ for oblate spheroids. This definition coincides with that using the circumscribing sphere. The light-scattering computations were performed specifically for this study, using the $T$-matrix code by [29] and assuming a refractive index $m=1.6+0.0005 i$. In this method, orientation averaging is analytic.

\subsection{Polyhedral prisms}

Polyhedral prisms are cylinders with a regular polygonal cross section. In this study, we consider prisms with square, pentagonal, hexagonal, heptagonal, and octagonal cross sections, i.e., polygonal cross sections with $N=4, \ldots, 8$ corners. Let $a$ be the distance from the centre of the polygon to any of its corners. Let further $h$ denote the length of the prism, i.e. the cylinder height. We then define the aspect ratio of the prism by $\epsilon=2 a / h$. The aspect ratio is smaller than unity for columns (or prolate prisms), and 
larger than unity for plates (or oblate prisms). We define the size parameter $x$ as $x=k h / 2$ for prolate and $x=k a$ for oblate prisms. As in the case of spheroids, we consider 21 different aspect ratios in the range between 1/3 and 3 , and use a refractive index of $m=1.6+0.0005 i$. The computations were carried out specifically for this study, using the TSYM code by [30]. In this method also the orientation averaging is analytic.

\subsection{Volcanic ash}

The volcanic ash particles reviewed in this study are examples of internally porous shapes, and are therefore called vesicular. Both particle types considered include cavities of varying sizes: for large-vesicle particles, the cavities create cratery features on the surface, whereas the structure of small-vesicle particles is more fluffy. The shapes are generated essentially by building a concave-hull-transformed cluster of spheres, where the spheres are replaced with voids shaped like Gaussian random spheres. The resulting particle surface is smoothed several times in a process similar to that described for flake particles, and possible loose small parts that are not in contact with the particle are removed. Shape generation, as well as the DDA-based light-scattering simulations for the shapes, are explained in more detail and with illustrations in [12]. Volcanic ash is typically composed of mainly glassy silicate with possible inhomogeneities that are more absorbing. Therefore, the refractive index has been set to $1.55+0.002 i$. For the large-vesicle ash, volume-equivalent size is assumed. For the small-vesicle ash, the size parameter describes a volume-equivalent size that an internally compact version of the small-vesicle particles would have (volume of the porous cavities are included in the volume), meaning that the actual volume-equivalent size pa- 
rameter would be somewhat smaller. For the scattering computations, 2925 orientations and ensembles of 5 particles were used for both shapes.

\subsection{Mie spheres}

We also show results for isotropic, homogeneous spheres obtained from the Mie theory. These are called Mie spheres for brevity. Mie spheres are still commonly used as an approximation for different nonspherical particle types in climate models and in many satellite retrieval algorithms. Here they are used for illustrating the differences in scattering by spherical and nonspherical particles. The spherical shape differs from other shapes considered here in that it does not vary from particle to particle in an ensemble and is also perfectly rotationally symmetric, so ensemble- and orientation averaging cannot be used to reduce the intrinsic interference patterns. We have thus used a narrow size distribution in the scattering computations: lognormal size distribution with a geometric standard deviation of 0.2 has been adapted, and radii within $\pm 1 \%$ of the geometric mean radius $r_{g}$ have been included. The size parameter has been defined in terms of $r_{g}$. For the refractive index, $m=1.6+0.001 i$ has been used. The light-scattering computations have been done specifically for this study, using the Mie code described in [24, page 158].

\subsection{Cluster of spheres}

In addition to single spheres, we consider clusters of ten equal-sized and homogeneous spheres. The clusters are formed by using a ballistic-clusteraggregation (BCA) method, where the positions of the spheres are determined by colliding spheres from random directions and attaching them at 
first collision. Overlapping between the spheres was not permitted. This method typically produces very fluffy clusters. To obtain packing densities comparable to those of the other particles in the study, the BCA method has been modified such that, for every ten collisions, only the sphere that collides closest to the first sphere of the cluster is attached. This results in denser clusters with a packing density that ranges from 0.20 to 0.34 . This method was also used in [12] for creating large-vesicle volcanic ash particles. For the individual spheres, we have chosen the refractive index of $m=1.6+0.0005 i$. The size parameter of the clusters is that of an equal-volume sphere. For each size parameter, ten random samples are used in the ensemble averaging, while orientation averaging is conducted analytically. The computations have been done specifically for this study, using the superposition $T$-matrix method by [31].

\section{Results}

As noted in the previous section, light-scattering data for different classes of geometries are difficult to compare directly, since it is not straightforward to define a common measure of size-equivalence for nonspherical particles. The data are also available only for relatively sparse set of discrete size parameters, so any attempt to convert the data to a (however defined) common measure of size equivalence would require considerable interpolation, and the comparison would depend on the common size equivalence adapted. We thus avoid direct shape-to-shape comparisons for specific sizes or size distributions but rather focus on comparing the size dependencies and generic features. Most of our analyses are qualitative rather than quantitative. 
The single-scattering properties of interest are shown in Figs. 2-7. Figures 2-5 show the scattering matrix elements, one shape per panel. For convenience, the panels are positioned the same way as the corresponding shapes in Fig. 1. Figs. 6 and 7 summarize other properties of interest.

\section{1. $S_{11}$}

The angular dependence of scattered intensity for unpolarized incident light, $S_{11}$, is shown in Fig. 2 for all the shapes considered. From the figure, it is immediately obvious that for most of the shapes considered, $S_{11}$ elements are qualitatively very similar with flat side-scattering angles, modest increase close the backscattering angles, nearly monotonic increase with increasing size parameter, and a general lack of distinct features at any scattering angle. The only clear exceptions are the Mie spheres and, to a lesser extend, the rough spheres, for which the interference patterns are quite pronounced. Many of the nonspherical shapes considered also show a weak secondary diffraction maximum at large size parameters close to $15-25^{\circ}$ scattering angle. This seems to be connected to the aspect ratio, as the feature is most pronounced for all nearly equi-dimensional shapes, while it is only weakly pronounced or entirely absent for the more elongated target types (both GRS and flake types, spheroids, and prisms).

For most nonspherical particles, especially towards larger size parameters, the side scattering becomes remarkably neutral. In contrast, side scattering for Mie spheres displays characteristic interference patterns and does not show a clear tendency for flattening within the size parameter range considered. There are, however, some modest differences also among the nonspherical particle types. For example, the angle where the minimum intensity is 
obtained varies considerably. For debris of spheres it is often found around $\theta=100^{\circ}$ scattering angle, while for Gaussian random spheres, agglomerated debris, flakes, and spheroids it is often much closer to backscattering direction. Thus, for some shapes, $S_{11}$ decreases however slightly but more or less monotonically almost to the backscattering direction, for some there is a minimum at side-scattering angles, and for some $S_{11}$ is nearly constant over a wide range of scattering angles.

Finally, all shapes produce some kind of backscattering enhancement. Again, there are some differences in details among the shapes. For example, the effect is very weak and broad for volcanic ash with small vesicles and for rough Gaussian random spheres. The Mie spheres produce a strong and broad enhancement toward backscattering especially for larger size parameters. For the cluster of spheres and rough spheres, the enhancement is moderately strong and broad.

\section{2. $-S_{12} / S_{11}$}

Compared to $S_{11}$, the degree of linear polarization for unpolarized incident light, $-S_{12} / S_{11}$, varies considerably among shapes (Fig. 3). There is also more angular variability in the values, albeit the rough-surface spheres and especially the Mie spheres again dominate in this respect.

Overall, the $-S_{12} / S_{11}$ values are more positive than negative for all the nonspherical shapes, while for the Mie spheres they are predominantly negative. For agglomerate debris particles, pocked spheres, and volcanic ash particles with both small and large vesicles, the values are always positive at side-scattering angles, and for rough Gaussian random spheres and damaged spheres nearly so. Only the Mie spheres, rough-surface spheres, and the 
flakes at smaller size parameters show negative values in this angular range. Each shape produces slightly negative polarization near forward-scattering angles, at least for some size parameters. Likewise, polarization tends to be negative close to backscattering.

The occurrence of interference patterns also varies among shapes. For Mie spheres they are very strong despite our effort to suppress them by averaging over a modest range of sizes. Obviously, a much broader size distribution would be needed to considerably dampen the interference patterns, which in turn would make the comparisons with the monodisperse nonspherical cases questionable. Debris of spheres, Gaussian random spheres, strongly damaged spheres, rough-surface spheres, and prisms also exhibit some interference, while for rough Gaussian spheres, small-vesicle ash, and agglomerated debris particles it is practically absent.

For most nonspherical shapes, $-S_{12} / S_{11}$ tends toward zero values with increasing size parameter. Polarization decreases with size nearly monotonously for agglomerated debris particles, rough Gaussian random spheres, and volcanic ash with small vesicles. Other shapes show varying deviations from this behavior, but for all shapes except Mie spheres the degree of polarization tends to be smaller for large than for small size parameters especially at side-scattering angles.

Small-scale surface roughness and internal inhomogeneity were identified by [28] as possible candidates to explain the observed bell-shaped positive polarization of laboratory-measured dust particle samples. Of the shapes considered here, only the agglomerated debris particles, small-vesicle ash, and to a lesser extend, the rough GRS particles, show such polarization 
characteristics throughout the size parameter range investigated. However, if scattering were averaged over a size distribution, several other particle types would probably also show similar polarization; for example, large-vesicle ash, pocked spheres, and probably those whose polarization is nearly zero for larger size parameters (e.g., damaged spheres, prisms). For the latter cases, the positive Rayleigh-like polarization from smaller size parameters might suffice to give the size-integrated polarization the desired angular dependence. Further, [32] show that both calcite and clay flakes would have such $-S_{12} / S_{11}$ after size integration. These results imply that surface roughness or inhomogeneity may not be needed to explain the measured polarization of mineral dust particles, although they definitely seem to promote such angular profile of polarization.

\section{3. $1-S_{22} / S_{11}$}

Similarly to the linear polarization, the depolarization ratio $D=1-$ $S_{22} / S_{11}$ shows larger particle-to-particle differences than the scattered intensity.

In general, $D$ tends to increase with increasing size parameter. This increase is monotonic for pocked spheres, rough Gaussian random spheres, and volcanic ash with both large and small vesicles; clearly non-monotonic it is only for both flakes, and even for them the overall tendency to increase with increasing size is clear. For Mie spheres, $D$ is identically zero.

The maximum $D$ values obtained for different shapes vary in a way that is clearly not due to different size equivalences. For example, both flakes produce maximum $D$ values of only about 0.6 , although their size is based on equivalent volume and, as very elongated shapes, their circumscribing 
sphere size equivalence would go up to about 20. Many shapes with smaller circumscribing sphere size equivalence nevertheless produce larger maximum $D$ values. Interestingly, the maximum $D$ for GRS particles is larger than that for rough GRS particles, although the latter can be considered to deviate more from the spherical shape. Likewise, small-vesicle ash particles depolarize weaker than the large-vesicle particles, consistently with similar findings by [33]. These findings might suggest that large-scale features are more important for depolarization than small-scale features. Then again, in the latter case, volcanic ash particles with small vesicles have much larger number of porous cavities than those with large vesicles, meaning that the internal field will be much more distorted and weaker. This could explain the difference between the two volcanic ash types.

There are also some differences at small scattering angles, for example, in how quickly $D$ deviates from zero as $\theta$ increases. For many shapes, $D$ starts to deviate from zero almost immediately outside the forward scattering direction, but for both GRS particles and, to a lesser extent, spheroids, it remains close to zero much further out. Second, both flakes show an interesting feature in $D$ that is not seen in any other shapes: at small size parameters $D$ is clearly non-zero at the forward-scattering angle of $\theta=0^{\circ}$. In [28], it was shown that this feature is seen also for very flat oblate spheroids. It thus seems to be a feature associated with very large aspect ratios. It might be useful for detecting the presence of thin, plate-like particles using forward scattering of linearly polarized light.

Almost all shapes produce a minimum in $D$ around $\theta=160^{\circ}$, moving closer to the backscattering direction with increasing $x$. This gives rise to a 
so-called double-lobe feature that is visible in all shapes except clay flakes. The double-lobe feature is most pronounced for the GRS particles, but the introduction of surface roughness (rough GRS) clearly weaken the second maximum at the exact backscattering direction. For most other nonspherical shapes, the second lobe is weaker than the first, but for spheroids, the second lobe is stronger.

In general, it appears difficult to draw any definite conclusions on how different types of nonspherical particles depolarize. For example, $D$ is very similar for the pocked spheres, agglomerated debris particles, and rough GRS particles, although these shapes are morphologically very different.

\section{4. $S_{33} / S_{11}-S_{44} / S_{11}$}

Figure 5 shows the values of $R=S_{33} / S_{11}-S_{44} / S_{11}$ for the model shapes considered. The obvious first observation is that near forward scattering direction $R$ tends to be very close to zero, and then become more negative towards backscattering directions, albeit not systematically. In general, $R$ also tends to become more negative with increasing $x$. The only clear exceptions are the Mie spheres, for which $R$ is identically zero and thus excluded from further analysis, and the two flakes. Except for the rough-surface spheres, largest negative values are obtained for the largest particles at the exact backscattering direction $\left(\theta=180^{\circ}\right)$.

The double-lobe feature in $D$ appears also in $R$, here as a local maximum at $\theta \approx 160^{\circ}$. This feature is seen for all shapes, but its exact location varies among shapes, and seems to move towards backscattering direction as the particle size increases, again in accordance with the double-lobe feature in $D$. The features are thus very likely to be related. 
Overall, the general angular dependence of $R$ for the shapes considered are similar, except for the flakes. In addition, rough-surface spheres show, again, strong interference patterns, but even for it the overall form is similar. There are, however, some possibly useful differences visible. For example, both rough GRS and small-vesicle ash particles produce much smaller negative $R$ values than most of the other shapes. Comparison with the similar smooth GRS particles and volcanic ash with large vesicles, respectively, implies that small-scale internal inhomogeneity and rough surface might lead to smaller deviations of $R$ from zero. A more detailed look on this is, however, in order to find out if this really is a generic result or just a coincidence.

Another potential morphological detail that could be identified from $R$ is the elongation of the particle, as strongly-elongated flakes show considerably different dependence of $R$ on $x$ and $\theta$. Further, $R$ might also be useful for detecting birefringent targets, as the differences between the calcite and clay flakes are considerable. These two cases have identical shapes and differ only by calcite flakes being birefringent. On the downside, measuring $R$ requires in practice that the whole $4 \times 4$ scattering matrix is measured.

\subsection{Other quantities}

Other quantities of interest are summarized in Figs. 6 and 7 as a function of size parameter $x$. As these plots compare differently shaped particles directly with each other, it is important to remember that their sizes are based on different size equivalences. Thus, $x$ of one shape is not necessarily comparable with $x$ of some other shape. Also, we note that the size parameter range for different shapes vary such that only size parameters from 4 to 10 have been computed for all shapes. Finally, it is noted that the refractive 
indices vary somewhat among the shapes. The plots should therefore be interpreted with caution.

The backscattered intensity $S_{11}\left(180^{\circ}\right)$ is shown in the top left panel of Fig. 6. The first obvious observation is that $S_{11}\left(180^{\circ}\right)$ tends to increase with increasing $x$, although this increase is not necessarily monotonic for individual shapes. Second, spheres tend to backscatter much more than the other shapes considered. Among the non-spherical shapes, clusters of spheres and rough spheres tend to be among the strongest backscatterers and the GRS and rough GRS particles among the weakest. These findings suggest that spherical particles tend to backscatter more efficiently than nonspherical, and that surface roughness reduces backscattering. The latter finding is consistent with the results obtained by [9]. A significant spread of about two orders of magnitude is seen among the shapes, which cannot be attributed solely on different size equivalences and slightly different $m$.

The maximum positive polarization, $\max \left(-S_{12} / S_{11}\right)$, is shown in the top right panel of Fig. 6. This quantity generally decreases from the unity of the Rayleigh scattering as $x$ increases, but for Mie spheres the behavior is rather opposite: the small values are obtained at small $x$ and large values at large $x$. At least partially this odd behavior is because at $x=2$, Mie scattering is already very non-Rayleigh-like with largely negative $-S_{12} / S_{11}$. The large positive values at larger $x$ are produced by the strong resonance peaks and do not indicate that polarization would be predominantly positive. The nonspherical shapes do not give rise to such strong resonances, which is at least partially because of the ensemble- and orientation averaging, and their $\max \left(-S_{12} / S_{11}\right)$ values are generally very different to those for Mie spheres. 
If Mie spheres are excluded, the spread among the shapes decreases as $x$ increases. Among the nonspherical shapes, the smallest values are obtained for the prisms, while many shapes compete for the largest. Overall, the volcanic ash with small vesicles is often found among those with largest $\max \left(-S_{12} / S_{11}\right)$.

The bottom left panel of Fig. 6 shows the minimum of linear polarization, $\min \left(-S_{12} / S_{11}\right)$, within the scattering angle range from $135^{\circ}$ to $180^{\circ}$. This is a proxy for the amplitude of the negative polarization branch (NPB). It is seen that, again, Mie spheres deviate strongly from the nonspherical shapes, producing consistently large negative values. Among the nonspherical shapes, the rough spheres and, at large size parameters, clusters of spheres produce the largest negative values and thus resemble most those for Mie spheres. The general trend among the other nonspherical shapes is a modest increase in the negative amplitude with increasing $x$ until, around $x=6-8$, the trend seems to reverse and a slight decrease with increasing $x$ is observed.

The scattering angle where the maximum positive polarization is achieved is presented in the bottom right panel of Fig. 6. In general, the maximum is located at side scattering angles, with a slight tendency towards larger scattering angles with increasing $x$. However, for size parameters $x \geq 6$, the maximum may also be located close to forward scattering angles for some shapes.

The top left panel of Fig. 7 shows the asymmetry parameter $g$ for each shape as a function of size parameter $x$. In general, $g$ tends to peak around $x \sim 6-8$. The spread among the shapes is also largest at those sizes. However, part of the spread may be artificial, caused by the different size equivalences 
used for different shapes. Of the shapes considered, the calcite and clay flakes tend to have the highest asymmetry parameters. For Mie spheres, and to a lesser extent for rough spheres, $g$ varies strongly from size to size. Overall, the spread among the shapes is considerable, despite the apparent similarity of the corresponding phase functions. There are some indications that for small $x$ the Mie spheres have smaller $g$ than nonspherical shapes, while for larger $x$ the situation appears to reverse. This behavior has been formerly noted by, e.g., [34, 35].

The linear depolarization at backscattering, $D\left(180^{\circ}\right)$, is presented at the top right panel of Fig. 7. For Mie spheres, this quantity is zero, while for nonspherical particles it usually deviates from it. The shapes considered here generally show an increasing trend in $D\left(180^{\circ}\right)$ as a function of $x$, but there are indications that $D$ plateaus for larger size parameters. The rough spheres usually produce the weakest depolarization (smallest $D$ ), while the strongest depolarizer varies among sizes. The spread among the shapes is quite considerable, but again this may be partially because of the different size equivalences among the shapes.

The linear polarization ratio at backscattering, $\mu_{L}\left(180^{\circ}\right)$, shown in the bottom left panel of Fig. 7 , is almost identical to $D\left(180^{\circ}\right)$ except that values of the latter are systematically somewhat larger. The explanation is that $S_{12}$ is identically zero at backscattering, and hence the definitions of these two quantities vary only for the denominator $\left(S_{11}\right.$ for $D$ and $S_{11}+S_{22}$ for $\left.\mu_{L}\right)$.

The circular polarization ratio at backscattering, $\mu_{C}\left(180^{\circ}\right)$, is shown in the bottom right panel of Fig. 7. Even though its definition differs considerably from that of $D$ and $\mu_{L}$, the results are qualitatively very similar 
with these quantities. The absolute values are different; note that $\mu_{C}$ is not bound between zero and unity. For spheres this quantity is zero because $P_{11}\left(180^{\circ}\right)=-P_{44}\left(180^{\circ}\right)$ (see e.g. [36], Table II).

\section{Summary and conclusions}

It is well established that scattering matrices of single particles depend on their shape, and that nonspherical particles scatter differently to spherical particles even after ensemble and orientation averaging. In contrast, possible differences or similarities in the scattering properties of different types of nonspherical particles have received little attention. To address this, we compared light-scattering properties of 15 different types of wavelength-scale particles as a function of size parameter. For all nonspherical particle types, the scattering matrices were ensemble- and orientation-averaged. For many of the particle types considered, previously published light-scattering data were used, resulting in small differences in the refractive indices and varying definitions of particle size.

The comparison reveals that the scattered intensity for unpolarized incident light, $S_{11}$, is generally very similar for the nonspherical particle types considered. There are some differences, but in each case the intensity distributions tend to be smooth, flat at the side scattering angles, and show at least a modest increase close to the exact backscattering angle. For

spheres, $S_{11}$ is clearly different, showing stronger interference structures and more pronounced backscattering enhancement. The asymmetry parameter, on the other hand, showed differences among the particle types that exceed differences that could be attributed to varying size equivalences and 
slightly different refractive indices of the shapes. This implies that, despite of the qualitative similarities, there are quantitative differences in the phase functions. The analysis of backscattered intensity showed that nonspherical shapes backscatter generally much less efficiently than the Mie spheres, and surface roughness was found to reduce backscattering further.

The degree of linear polarization for incident unpolarized light, $-S_{12} / S_{11}$, on the other hand, shows clear differences among the particle types, but there are also similarities. For example, $-S_{12} / S_{11}$ tends to be much more positive for nonspherical particles than for Mie spheres. Likewise, for all nonspherical shapes, the absolute value of the degree of polarization tends to decrease considerably with increasing size. Although only few of the shapes considered produced bell-like positive polarization curves for all size parameters considered, quite a few of them could have shown that kind of polarization if averaged over a size distribution. Thus, even though small-scale surface roughness and internal inhomogeneity promote this type of polarization and has been offered as an explanation for such polarization in laboratorymeasured polarization curves for mineral dust samples, other explanations are clearly also possible. Polarization patterns and particle morphologies did not correlate very clearly, as some seemingly quite different shapes produced very similar angular distribution.

Similar to the $S_{11}$ element, the depolarization ratios $1-S_{22} / S_{11}$ tended to be fairly similar for the particle types considered, except for the Mie spheres for which it is identically zero. One clear exception among the nonspherical shapes were the platy flake particles, suggesting that high-aspect-ratio particles depolarize differently to more equidimensional nonspherical parti- 
cles. More interference patterns were seen for particles that do not have small-scale surface roughness or small-scale internal inhomogeneity. Close to a scattering angle $\theta=160^{\circ}$ an interesting double-lobe feature was seen that is present for almost all particle types considered but nevertheless often differs in details. Interestingly, there are some differences between the model particles in how quickly $D$ starts to deviate from zero as the scattering angle increases from $0^{\circ}$. For example, according to laboratory measurements $[37,38], D$ for many mineral particle samples starts to deviate from zero almost immediately. This is one aspect where distributions of spheroids clearly fail when mimicking scattering by these samples $[13,17,39]$. In general, the dependence of $D$ on the particle morphology seems to defy simplistic explanations. Thus, even though $D$ is a very useful indicator for deviations from particle isotropy (due to nonspherical shape, for example), it does not seem particularly useful for quantifying this deviation, or for identifying different classes of particle morphology.

The dependence of $S_{33} / S_{11}-S_{44} / S_{11}$ on the scattering angle was generally similar for most particle types considered, with the notable exception of spheres. Again, the high-aspect ratio flake particles showed the largest deviations from the other nonspherical particles. This is especially true for the birefringent calcite flakes, indicating that this quantity might be useful for detecting birefringent species. On the other hand, it does not seem promising for differentiating other types of nonsphericity. For Mie spheres $S_{33} / S_{11}-S_{44} / S_{11}$ is identically zero, so it is another quantity that could be used for identifying deviations from isotropy.

Overall, it seems that, qualitatively, different types of nonspherical par- 
ticles scatter light surprisingly similarly. This implies that scattering by nonspherical particles can often be mimicked by other types of nonspherical model particles. Still, there are indications that morphological features such as the degree of surface roughness or the size-scale of internal structure could very well have systematic effects on scattering. Further, there are clearly quantitative differences in scattering by different shapes. Thus, despite great similarities, ensembles of different shapes cannot be considered equal. Of the parameters considered here, the degree of linear polarization for incident unpolarized light seems most promising for inverting particle shapes. Luckily, it is also one of the parameters that can be measured for targets illuminated by natural light and thus applies to passive remote sensing. Shape inversion based on light scattering seems nevertheless difficult, in particular because light-scattering properties do not seem to correlate well with morphological details: seemingly similar particles may scatter differently and seemingly different very similarly. Finally, it is emphasized that the results shown here only apply to ensemble averages of randomly oriented nonspherical particles. Undoubtedly single nonspherical particles in fixed orientation can have very different and distinct single-scattering properties. Indeed, single-particle shape inversions would probably be much easier and might be potentially useful for deriving three-dimensional shapes of real nonspherical particles if such measurements could be carried out.

\section{Acknowledgments}

Timo Nousiainen, Hannakaisa Lindqvist and Evgenij Zubko acknowledge funding by the Academy of Finland (contracts 125180 and 127461). Michael 
Kahnert acknowledges funding from the Swedish Research Council under project 621-2008-4387 and 621-2011-3346. Michael Mishchenko and Dan Mackowski are acknowledged for making their light-scattering models publicly available. Finally, we want to thank the Finnish IT Center for Science (CSC) for the permission to use their superclusters.

\section{References}

[1] G. Mie, Beiträge zur Optik trüber Medien, speziell kolloidaler Metallösungen, Ann. Phys. 1908;25:377-445.

[2] M. I. Mishchenko, L. D. Travis, R. A. Kahn, R. A. West, Modeling phase functions for dustlike tropospheric aerosols using a shape mixture of randomly oriented polydisperse spheroids, J. Geophys. Res. 1997;102:16831-16847.

[3] M. Kahnert, Electromagnetic scattering by nonspherical particles: Recent advances, J. Quant. Spectrosc. Radiat. Transfer 2010;111:17881790.

[4] D. W. Mackowski, Calculation of total cross sections of multiple-sphere clusters, J. Opt. Soc. Am. A 11 1994;11:2851-2861.

[5] L. Liu, M. I. Mishchenko, W. P. Arnott, A study of radiative properties of fractal soot aggregates using the superposition $T$-matrix method, J. Quant. Spectrosc. Radiat. Transfer 109 2008;109:2656-2663.

[6] M. Kahnert, Numerically exact computation of the optical properties of 
light absorbing carbon aggregates for wavelength of $200 \mathrm{~nm}-12.2 \mu \mathrm{m}$, Atmos. Chem. Phys. 10 2010;10:8319-8329.

[7] K. Muinonen, T. Nousiainen, P. Fast, K. Lumme, J. I. Peltoniemi, Light scattering by Gaussian random particles: Ray optics approximation, J. Quant. Spectrosc. Radiat. Transfer 1996;55:577-601.

[8] T. Rother, K. Schmidt, J. Wauer, V. Shcherbakov, J.-F. Gayet, Light scattering on Chebyshev particles of higher order, Appl. Opt. 2006;45:6030-6037.

[9] M. Kahnert, T. Nousiainen, P. Mauno, On the impact of non-sphericity and small-scale surface roughness on the optical properties of hematite aerosols, J. Quant. Spectrosc. Radiat. Transfer 2011;112:1815-1824.

[10] M. Kahnert, T. Rother, Modeling optical properties of particles with small-scale surface roughness: combination of group theory with a perturbation approach, Opt. Express 2011;19:11138-11151.

[11] M. Mishchenko, J. Dlugach, D. Mackowski, Light scattering by wavelength-sized particles dusted with subwavelength-sized grains, Optics Letters 2011;36:337-339.

[12] H. Lindqvist, T. Nousiainen, E. Zubko, O. Muñoz, Optical modeling of vesicular volcanic ash particles, J. Quant. Spectrosc. Radiat. Transfer 2011;112:1871-1880. doi:10.1016/j.jqsrt.2011.01.032.

[13] S. Merikallio, H. Lindqvist, T. Nousiainen, M. Kahnert, Modelling light scattering by mineral dust using spheroids: assessment of applicability, 
Atmos. Chem. Phys. 11 (11) 2011;11:5347-5363. doi:10.5194/acp-11$5347-2011$.

URL http://www . atmos-chem-phys . net/11/5347/2011/

[14] M. I. Mishchenko, L. D. Travis, Light scattering by polydisperse, rotationally symmetric nonspherical particles: Linear polarization, J. Quant. Spectrosc. Radiat. Transfer 1994;51:759-778.

[15] P. Yang, K. N. Liou, K. Wyser, D. Mitchell, Parameterization of the scattering and absorption properties of individual ice crystals, J. Geophys. Res. 2000;105:4699-4718.

[16] Z. Meng, P. Yang, G. Kattawar, L. Bi, K. Liou, I. Laszlo, Singlescattering properties of tri-axial ellipsoidal mineral dust aerosols: A database for application to radiative transfer calculations, J. Aerosol Sci. 2010;41:501-512. doi:10.1016/j.jaerosci.2010.02.008.

[17] T. Nousiainen, M. Kahnert, B. Veihelmann, Light scattering modeling of small feldspar aerosol particles using polyhedral prisms and spheroids, J. Quant. Spectrosc. Radiat. Transfer 2006;101:471-487. doi:10.1016/j.jqsrt.2006.02.038.

[18] M. Kahnert, T. Nousiainen, M. A. Thomas, J. Tyynelä, Light scattering by particles with small-scale surface roughness: comparison of four classes of model geometries, J. Quant. Spectrosc. Radiat. Transfer (accepted).

[19] B. Veihelmann, T. Nousiainen, M. Kahnert, W. J. van der Zande, Light scattering by small feldspar particles simulated using the Gaus- 
sian random sphere geometry, J. Quant. Spectrosc. Radiat. Transfer 2006;100:393-405. doi:10.1016/j.jqsrt.2005.11.053.

[20] L. Bi, P. Yang, G. Kattawar, R. Kahn, Single-scattering properties of triaxial ellipsoidal particles for a size parameter range from the Rayleigh to geometric-optics regimes, Appl. Opt. 2009;48:114-126.

[21] L. Bi, P. Yang, G. Kattawar, R. Kahn, Modeling optical properties of mineral aerosol particles by using nonsymmetric hexahedra, Appl. Opt. 2010;49:334-342.

[22] T. Nousiainen, M. Kahnert, H. Lindqvist, Can particle shape information be retrieved from light-scattering observations using spheroidal model particles?, J. Quant. Spectrosc. Radiat. Transfer 2011;112:22132225. doi:10.1016/j.jqsrt.2011.05.008.

[23] C. F. Bohren, D. R. Huffman, Absorption and Scattering of Light by Small Particles, John Wiley \& Sons, New York, 1983, 530 pp.

[24] M. I. Mishchenko, , L. D. Travis, A. A. Lacis, Scattering, absorption, and emission of light by small particles, Cambridge, Cambridge University Press; 2002, 445 pp.

[25] E. Zubko, Y. Shkuratov, N. Kiselev, G. Videen, DDA simulations of light scattering by small irregular particles with various structure, J. Quant. Spectrosc. Radiat. Transfer 2006;101:416-434. doi:10.1016/j.jqsrt.2006.02.055. 
[26] E. Zubko, Light scattering by irregularly shaped particles with sizes comparable to the wavelength, Vol. 6 of Light Scattering Reviews, SpringerVerlag; 2011, Ch. 2, pp. 39-74.

[27] E. Zubko, K. Muinonen, Y. Shkuratov, G. Videen, T. Nousiainen, Scattering of light by roughened Gaussian random particles, J. Quant. Spectrosc. Radiat. Transfer 2007;106:604-615. doi:10.1016/j.jqsrt.2007.01.050.

[28] T. Nousiainen, E. Zubko, J. V. Niemi, K. Kupiainen, M. Lehtinen, K. Muinonen, G. Videen, Single-scattering modeling of thin, birefringent mineral dust flakes using the discrete-dipole approximation, J. Geophys. Res. 2009;114:D07207. doi:10.1029/2008JD011564.

[29] M. I. Mishchenko, L. D. Travis, Capabilities and limitations of a current Fortran implementation of the $T$-matrix method for randomly oriented rotationally symmetric scatterers, J. Quant. Spectrosc. Radiat. Transfer 1998;60:309-324.

[30] M. Kahnert, Irreducible representations of finite groups in the T matrix formulation of the electromagnetic scattering problem, J. Opt. Soc. Am. A 2005;22:1187-1199.

[31] D. Mackowski, M. Mishchenko, Calculation of the T matrix and the scattering matrix for ensembles of spheres, J. Opt. Soc. Amer. A 1996;13:2266-2278.

[32] T. Nousiainen, O. Muñoz, H. Lindqvist, P. Mauno, G. Videen, Light scattering by large Saharan dust particles: Comparison of modeling and 
experimental data for two samples, J. Quant. Spectrosc. Radiat. Transfer 2011;112:420-433. doi:10.1016/j.jqsrt.2010.09.003.

[33] H. Lindqvist, K. Muinonen, T. Nousiainen, Light scattering by coated Gaussian and aggregate particles, J. Quant. Spectrosc. Radiat. Transfer 2009;110:1398-1410. doi:10.1016/j.jqsrt.2009.01.015.

[34] M. Kahnert, T. Nousiainen, P. Räisänen, Mie simulations as an error source in mineral aerosol radiative forcing calculations, Quart. J. Roy. Meteorol. Soc. 2007;133:299-307. doi:doi:10.1002/qj.40.

[35] P. Mauno, P. Räisänen, M. Kahnert, T. Nousiainen, Sensitivity of the shortwave radiative effect of dust on particle shape: comparison of spheres and spheroids, J. Geophys. Res.(in press).

[36] J. Hovenier, C. van der Mee, Basic relationships for matrices describing scattering by small particles, in: M. I. Mishchenko, J. W. Hovenier, L. D. Travis (Eds.), Light Scattering by Nonspherical Particles, San Diego, Academic Press; 2000, Ch. 3, pp. 61-85.

[37] H. Volten, O. Muñoz, J. Hovenier, L. Waters, An update of the Amsterdam Light Scattering Database, J. Quant. Spectrosc. Radiat. Transfer 2006;100:437-443.

[38] O. Muñoz, H. Volten, Experimental light scattering matrices from the Amsterdam light scattering database, in: A. Kokhanovsky (Ed.), Light Scattering Reviews, Springer, 2006, pp. 3-30.

[39] T. Nousiainen, K. Vermeulen, Comparison of measured single-scattering 
matrix of feldspar particles with $T$-matrix simulations using spheroids, J. Quant. Spectrosc. Radiat. Transfer 2003;79-80:1031-1042. 


\section{List of Figures}

1 Example images of the shapes considered in the study. . . . . 37

2 The angular dependence of scattered intensity for unpolarized incident light, $S_{11}$ for all the shapes considered. The different line styles correspond to size parameters 2 (solid black), 4 (dashed black), 6 (dotted black), 8 (solid grey), 10 (dashed grey), and 12 (dotted grey). For the definition of the size equivalences for each shape, see Sect. 3. . . . . . . . . . . 38

3 As Fig. 2 but for $-S_{12} / S_{11}$, the degree of linear polarization for incident unpolarized light. . . . . . . . . . . . . . . . 39

$4 \quad$ As Fig. 2 but for the depolarization ratio $D=1-S_{22} / S_{11}$. . 40

$5 \quad$ As Fig. 2 but for $S_{33} / S_{11}-S_{44} / S_{11} \ldots \ldots \ldots$. . . . . . . 41

6 Dependence of $S_{11}(180)$ (top left panel), $\max \left(-S_{12} / S_{11}\right)$ (top right panel), $\min \left[-S_{12}(135: 180) / S_{11}(135: 180)\right]$ (bottom left panel), and the scattering angle of $\max \left(-S_{12} / S_{11}\right)$ (bottom right panel) as a function of size parameter (as defined for each shape). . . . . . . . . . . . . . . . . 42

7 As Fig. 6 but for the asymmetry parameter (top left panel), $D(180)$ (top right panel), $\mu_{L}$ (bottom left panel), and $\mu_{C}$ (bottom right panel). . . . . . . . . . . . . . . 43 


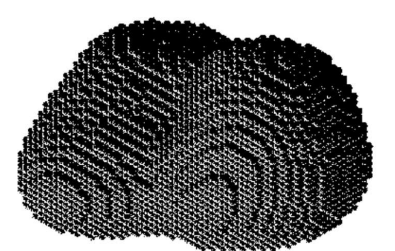

Gaussian random sphere (GRS)

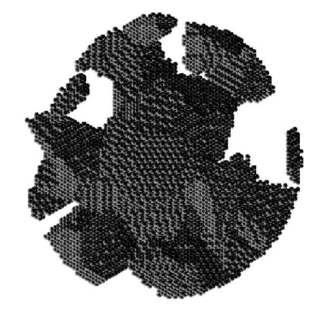

Pocked spheres

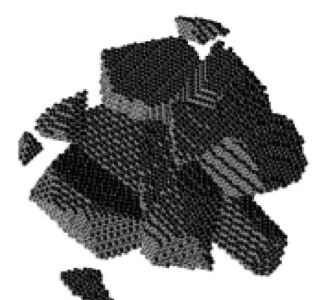

Agglomerated debris

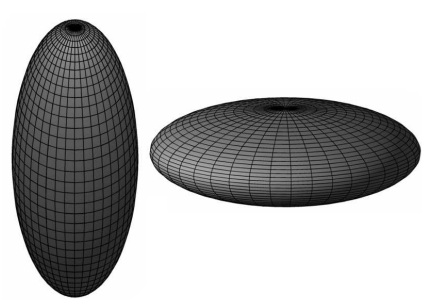

Spheroids

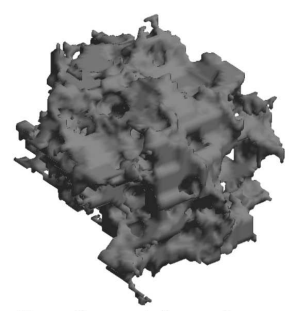

Small-vesicle ash

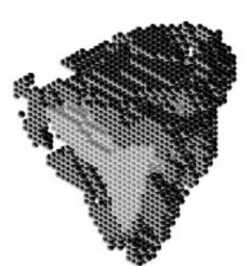

Rough GRS

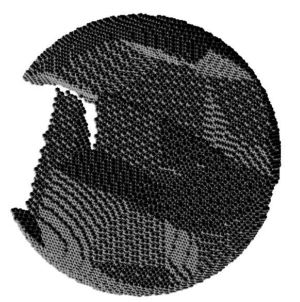

Damaged spheres

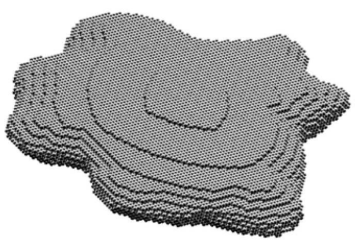

Calcite flakes

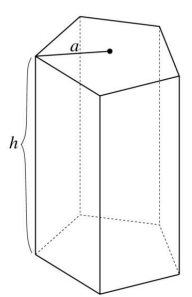

Prisms

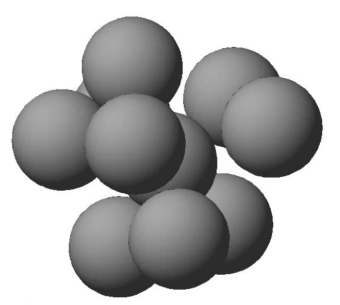

Cluster of spheres

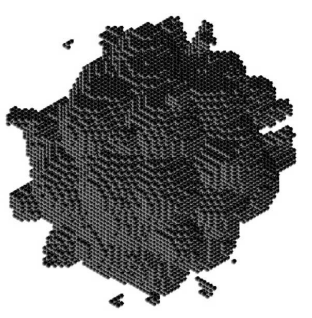

Rough spheres

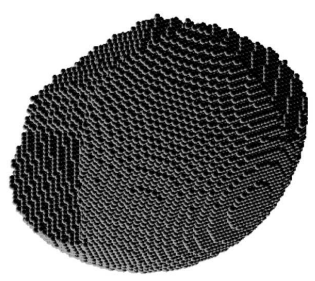

Debris of spheres

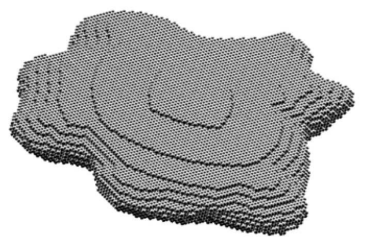

Clay flakes

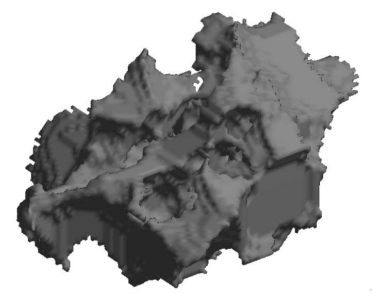

Large-vesicle ash

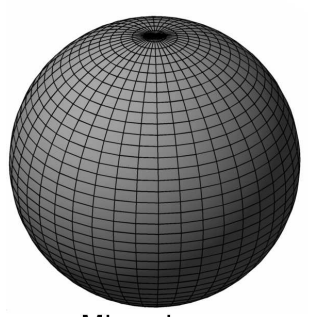

Mie spheres

Figure 1: Example images of the shapes considered in the study. 

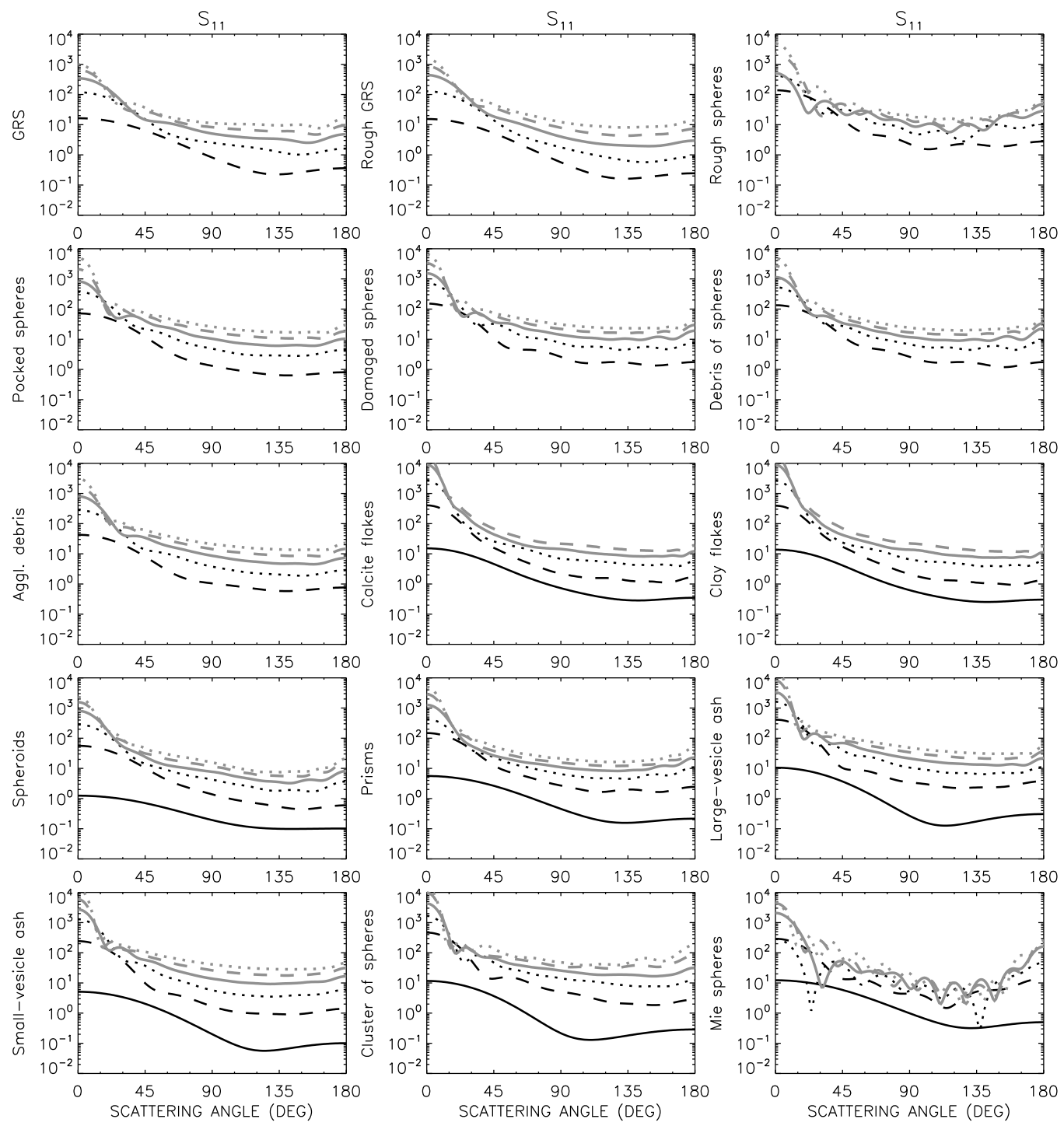

Figure 2: The angular dependence of scattered intensity for unpolarized incident light, $S_{11}$ for all the shapes considered. The different line styles correspond to size parameters 2 (solid black), 4 (dashed black), 6 (dotted black), 8 (solid grey), 10 (dashed grey), and 12 (dotted grey). For the definition of the size equivalences for each shape, see Sect. 3. 

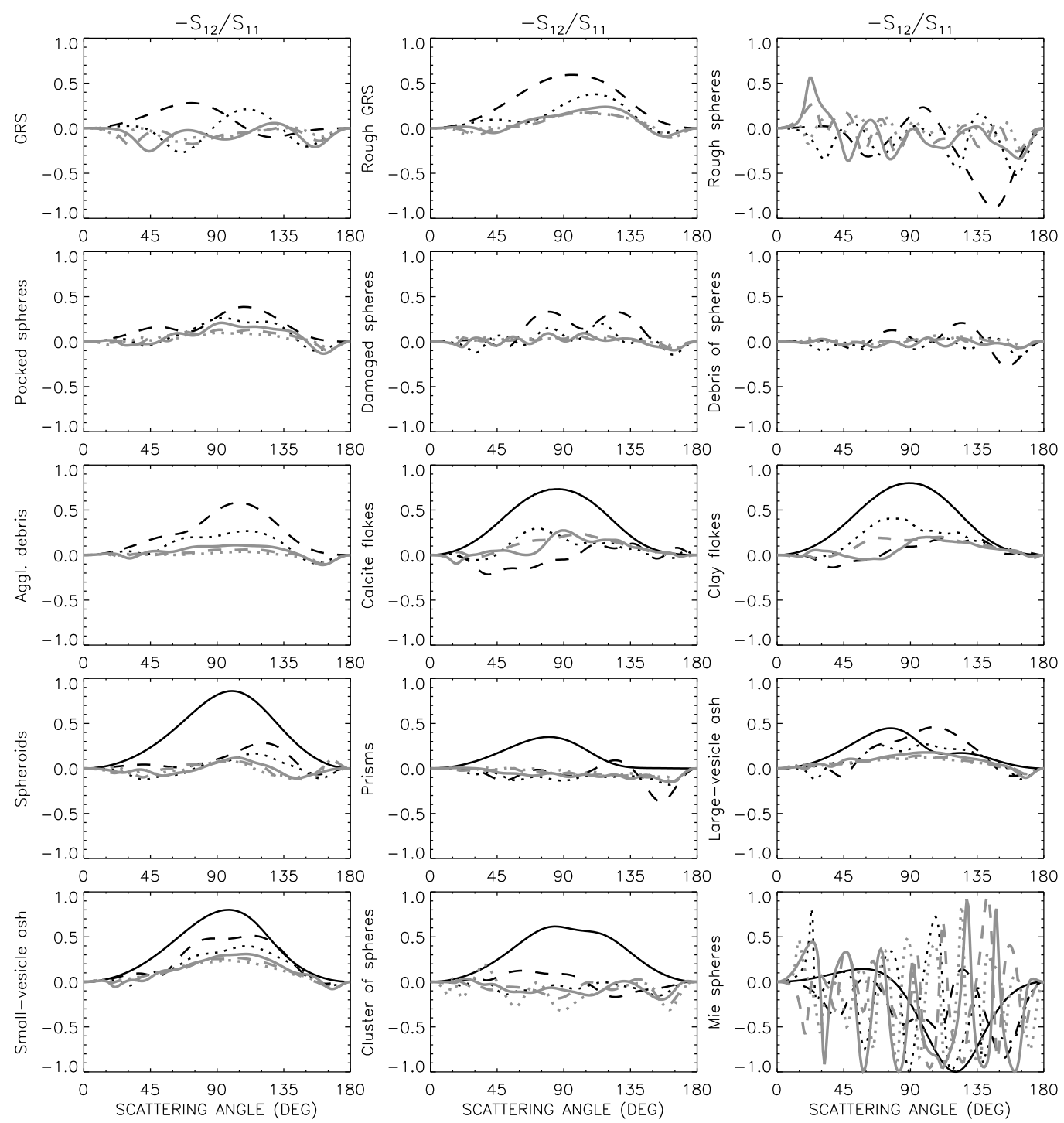

Figure 3: As Fig. 2 but for $-S_{12} / S_{11}$, the degree of linear polarization for incident unpolarized light. 

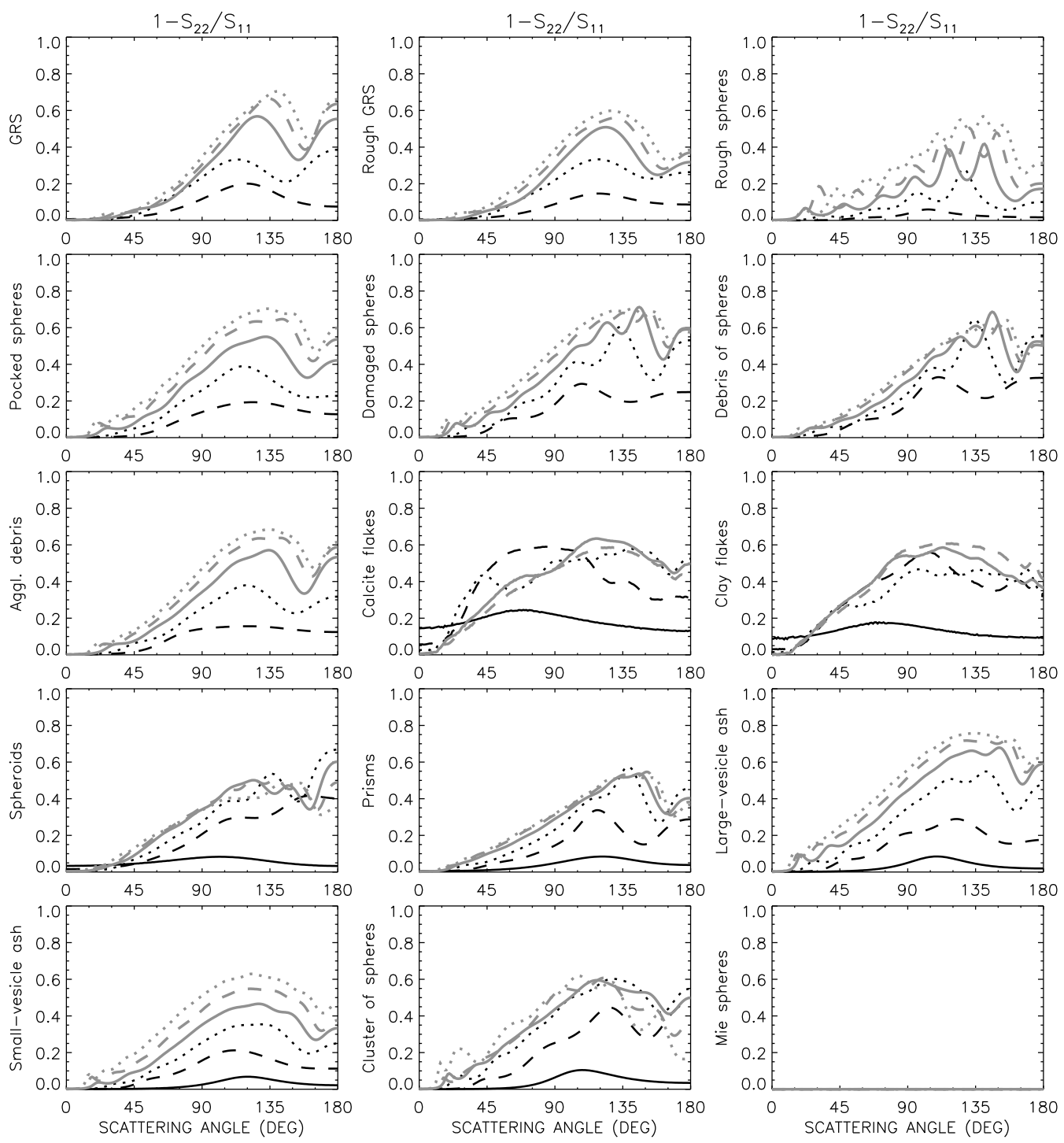

Figure 4: As Fig. 2 but for the depolarization ratio $D=1-S_{22} / S_{11}$. 

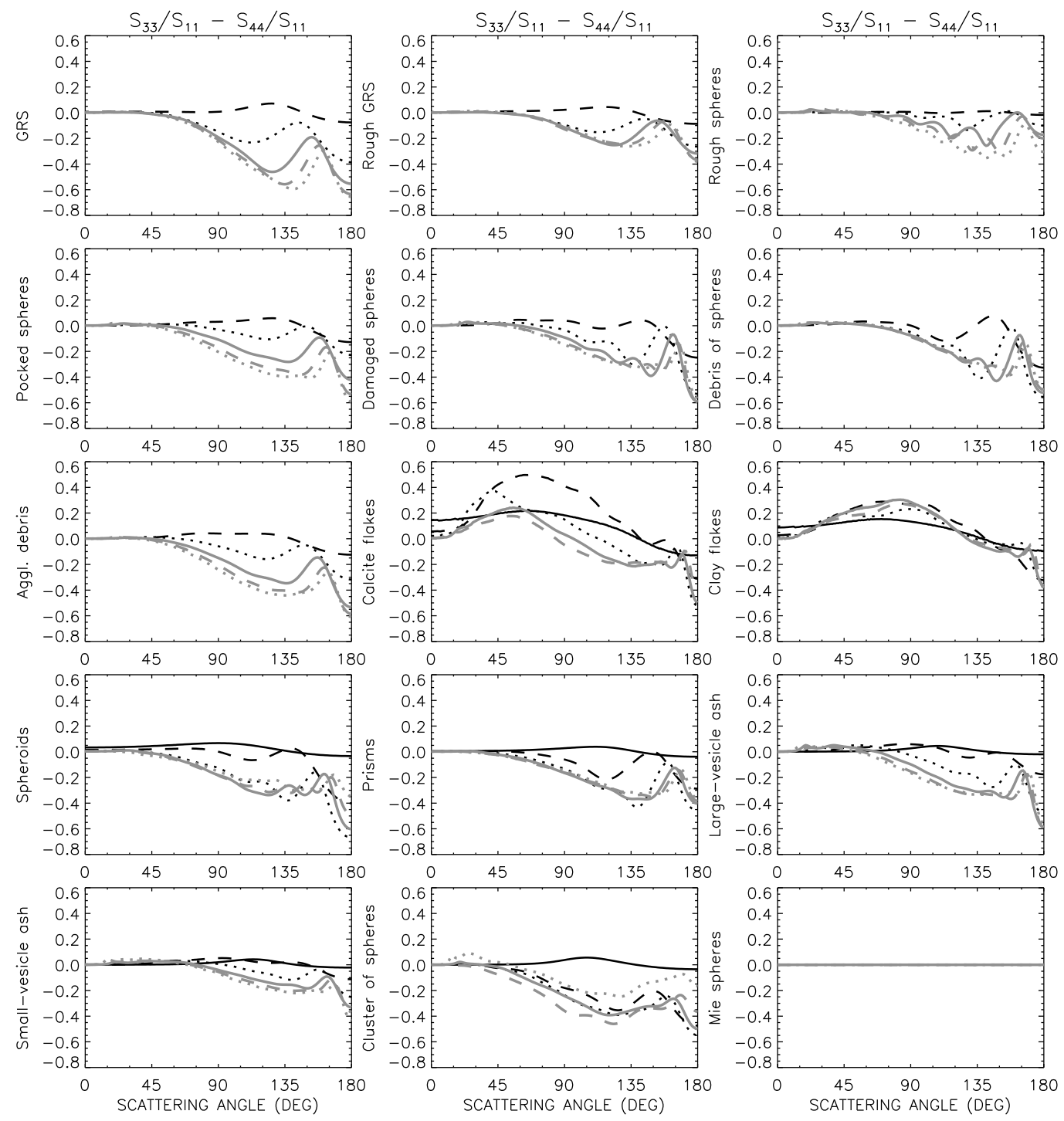

Figure 5: As Fig. 2 but for $S_{33} / S_{11}-S_{44} / S_{11}$. 

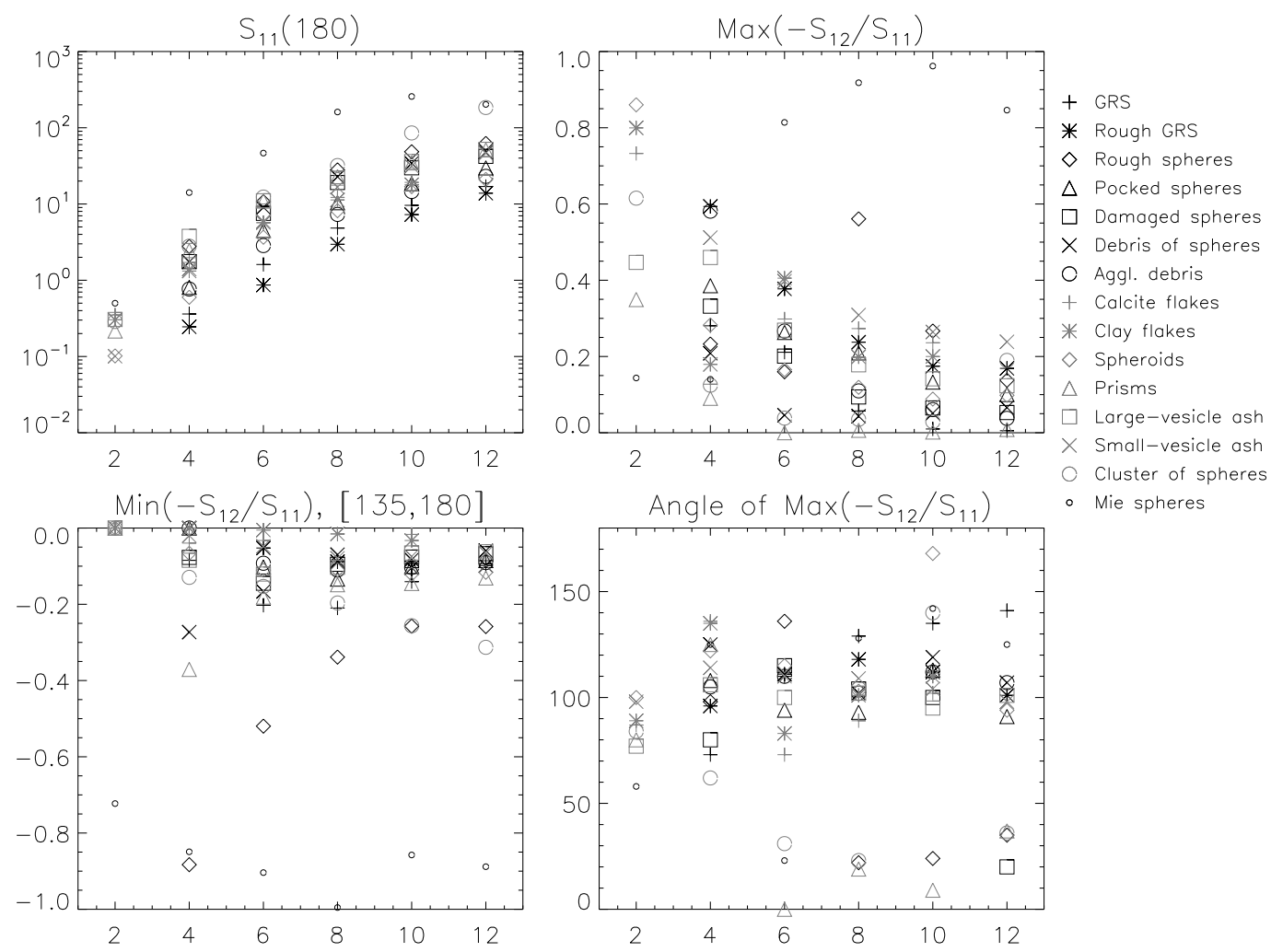

Figure 6: Dependence of $S_{11}(180)$ (top left panel), $\max \left(-S_{12} / S_{11}\right)$ (top right panel), $\min \left[-S_{12}(135: 180) / S_{11}(135: 180)\right]$ (bottom left panel), and the scattering angle of $\max \left(-S_{12} / S_{11}\right)$ (bottom right panel) as a function of size parameter (as defined for each shape). 

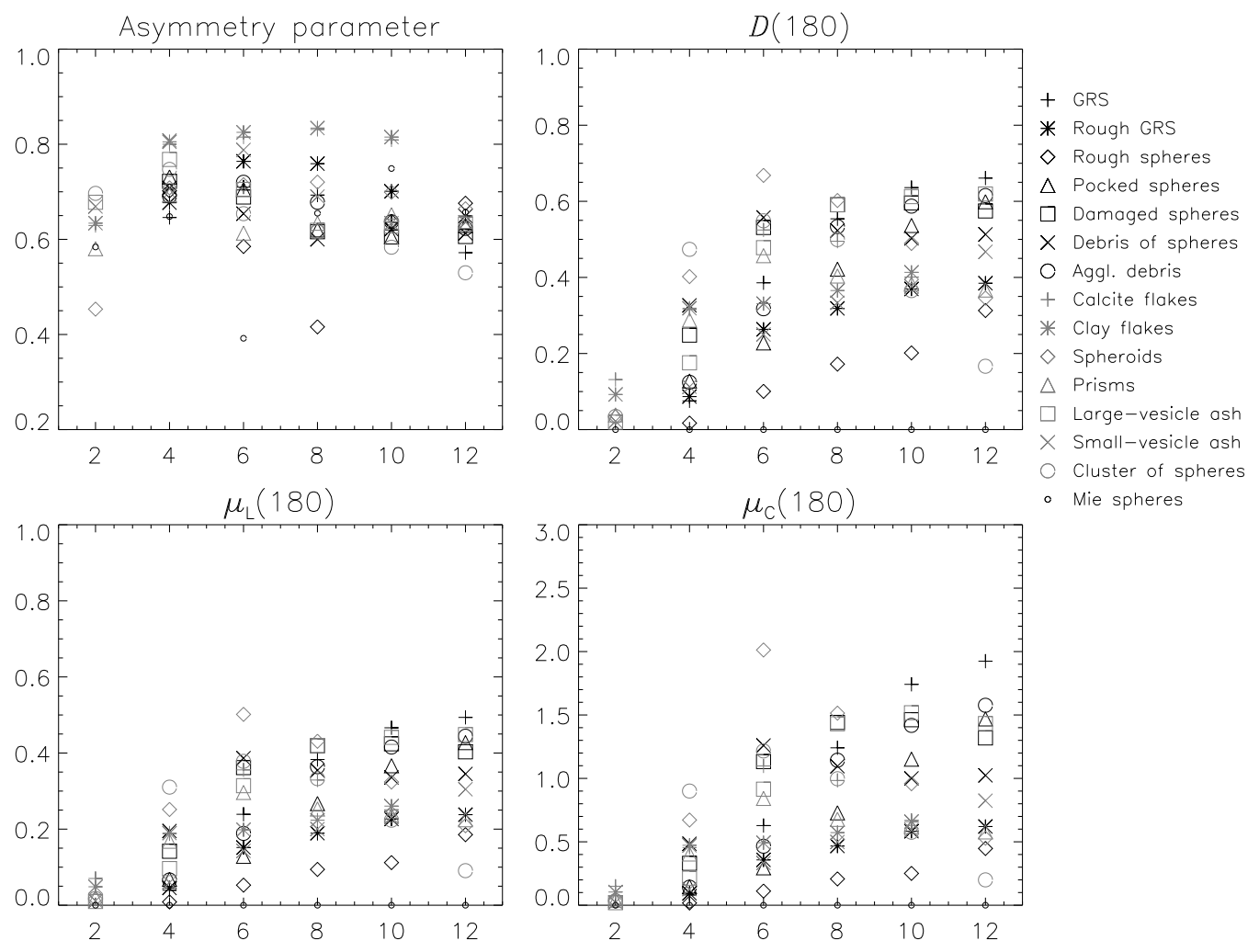

Figure 7: As Fig. 6 but for the asymmetry parameter (top left panel), $D(180)$ (top right panel), $\mu_{L}$ (bottom left panel), and $\mu_{C}$ (bottom right panel). 


\section{List of Tables}

1 Summary of the particle shapes considered. . . . . . . . . . . . 45 
Table 1: Summary of the particle shapes considered.

\begin{tabular}{lcccc}
\hline Shape & $m$ & $x$ & size equivalence & Reference \\
\hline \hline GRS & $1.6+0.0005 i$ & $4-12$ & circumscribing sphere & {$[27]$} \\
Rough GRS & $1.6+0.0005 i$ & $4-12$ & circumscribing sphere & {$[27]$} \\
Rough spheres & $1.6+0.0005 i$ & $4-12$ & zcircumscribing sphere & {$[25]$} \\
Pocked spheres & $1.6+0.0005 i$ & $4-12$ & circumscribing sphere & {$[25]$} \\
Damaged spheres & $1.6+0.0005 i$ & $4-12$ & circumscribing sphere & {$[25]$} \\
Debris of spheres & $1.6+0.0005 i$ & $4-12$ & circumscribing sphere & {$[26]$} \\
Aggl. debris & $1.6+0.0005 i$ & $4-12$ & circumscribing sphere & {$[25]$} \\
Calcite flakes & birefringent & $2-10$ & volume & {$[28]$} \\
Clay flakes & $1.603+0.0 i$ & $2-10$ & volume & {$[28]$} \\
Spheroids & $1.6+0.0005 i$ & $2-12$ & circumscribing sphere & - \\
Prisms & $1.6+0.0005 i$ & $2-12$ & length & - \\
Large-vesicle ash & $1.55+0.002 i$ & $2-12$ & volume & {$[12]$} \\
Small-vesicle ash & $1.55+0.002 i$ & $2-12$ & volume (inc. vesicles) & {$[12]$} \\
Cluster of spheres & $1.6+0.0005 i$ & $2-12$ & volume & - \\
Mie spheres & $1.6+0.001 i$ & $2-12$ & n.a. & - \\
\hline
\end{tabular}

